\title{
On the Nodal Count Statistics for Separable Systems in any Dimension
}

\author{
Sven Gnutzmann and Stylianos Lois \\ School of Mathematical Sciences, University of Nottingham, Nottingham NG7 2RD, \\ UK
}

\begin{abstract}
We consider the statistics of the number of nodal domains aka nodal counts for eigenfunctions of separable wave equations in arbitrary dimension. We give an explicit expression for the limiting distribution of normalised nodal counts and analyse some of its universal properties. Our results are illustrated by detailed discussion of simple examples and numerical nodal count distributions.
\end{abstract}




\section{Introduction}

We consider real square-integrable eigenfunctions $\Phi(\mathbf{q})$ of the stationary Schrödinger equation

$$
H \Phi(\mathbf{q}) \equiv-\Delta_{\mathcal{M}} \Phi(\mathbf{q})+V(\mathbf{q}) \Phi(\mathbf{q})=E \Phi(\mathbf{q})
$$

for a massive point particle on an $s$-dimensional smooth connected Riemannian manifold $\mathcal{M}$ with local coordinates $\mathbf{q} \equiv\left(q^{1}, \ldots, q^{s}\right)$. Here, $\Delta_{\mathcal{M}}$ is the Laplace-Beltrami operator on $\mathcal{M}, V(\mathbf{q})$ is a potential, and $E$ is an energy eigenvalue. We have set the value of the physical constant $\frac{\hbar^{2}}{2 m}$ of Planck's constant squared over twice the mass of the particle equal to one by appropriate choice of units.

We will allow that $\mathcal{M}$ has a boundary and will impose boundary conditions on $\Phi(\mathbf{q})$ such that the Schrödinger operator $H$ defined in (1) is self-adjoint (e.g. Dirichlet or Neumann boundary conditions).

We consider only non-negative potentials for which the classically allowed region $\mathcal{V}_{E}=\{\mathbf{q}: V(\mathbf{q}) \leq E\}$ is compact and connected. This ensures a discrete and nonnegative energy spectrum. If $V(\mathbf{q})=0$ (free motion) the condition implies that the manifold $\mathcal{M}$ is compact.

We arrange the spectrum in ascending order as $0 \leq E_{1}<E_{2} \leq \cdots \leq E_{N} \leq$ $E_{N+1} \leq \cdots$ and denote by $\Phi_{N}(\mathbf{q})$ the eigenfunction corresponding to $E_{N}$. For a given eigenfunction $\Phi_{N}(\mathbf{q})$ the nodal set $\mathcal{N}=\Phi_{N}^{-1}(0) \subset \mathcal{M}$ consists of all points on the manifold where the eigenfunction vanishes. A nodal domain $\mathcal{D} \subset \mathcal{M}$ of $\Phi_{N}(\mathbf{q})$ is a maximally connected region where the sign of $\Phi_{N}(\mathbf{q})$ does not change.

The characterisation of eigenfunctions in terms of their nodal set has a history which is more than 200 years old with the first systematic treatment by Chladni [1] who visualised the vibration modes of plates with sand that accumulates at the nodal set. Among other things he also counted the number of different nodal domains for each vibration mode and used these number to characterise the modes for a given shape. The number of nodal domains of eigenfunctions or nodal counts will also be the subject of the present contribution. For the wave function $\Phi_{N}$ we denote the nodal count by $\nu_{N}$. The collection of all nodal counts forms the nodal sequence $\left\{\nu_{N}\right\}_{N=1}^{\infty}$ (for systems with degenerate eigenvalues this definition of the nodal sequence is incomplete).

In one dimension Sturm's oscillation theorem [2] states $\nu_{N}=N$ under very general conditions. The generalisation of this seminal result to quasi one dimensional systems such as quantum graphs has been a recent research topic [3, 4]. For arbitrary dimension a seminal result is Courant's nodal domain theorem [5] which states $\nu_{N} \leq N$ for the Laplacian in any dimension. Pleijel later showed that in dimension $d=2$ the upper bound $\nu_{N}=N$ is achieved only a finite number of times [6].

In recent years it has been established that the nodal sequence contains a lot of information about the underlying geometry. It has been conjectured in [7] that the nodal count sequence in some cases allows a full reconstruction of the manifold $\mathcal{M}$ up to an overall scaling factor, and that it can be used to distinguish between isospectral 
partners. These conjectures have been partly confirmed and refined in recent years $[8,9,10,11,12,13]$.

Another recent line of research focusses on the statistics of the nodal counts. To this end one defines [14] the normalised nodal count by the ratio

$$
\xi_{N}=\frac{\nu_{N}}{N}
$$

and focusses on the distribution of its values in an energy window. Courant's theorem implies $0<\xi_{N} \leq 1$. For a given spectral interval $I_{g}(E)=[E,(1+g) E]($ where $g>0)$ one defines the nodal count distribution formally by

$$
P(\xi)_{I_{g}(E)}=\frac{1}{N_{I_{g}(E)}} \sum_{N: E_{N} \in I_{g}(E)} \delta\left(\xi-\xi_{N}\right)
$$

where $N_{I_{g}(E)}$ is the number of eigenvalues in $I_{g}(E)$. An interesting question concerns the existence and properties of a (smooth) limiting distribution

$$
P(\xi)=\lim _{E \rightarrow \infty} P(\xi)_{I_{g}(E)} .
$$

Such a limit may exist (in the weak sense) because the number of states in the interval $I_{g}(E)$ grows as $E \rightarrow \infty$.

For two-dimensional separable systems a semiclassical theory shows $[14,15]$ that the limiting function indeed exists and that it can be expressed explicitly in terms of the corresponding integrable classical dynamics. It has a number of universal features:

(i) The limiting distribution has support $0 \leq \xi<\xi_{\text {crit }}$ where the critical value is smaller than one (which is consistent with Pleijel's theorem [6]).

(ii) Near the critical value the limiting distribution has a square-root singularity

$$
P(\xi) \sim\left(\xi_{\text {crit }}-\xi\right)^{-1 / 2} \quad \text { for } \xi<\xi_{\text {crit }} .
$$

In this work we will generalize this theory to separable systems in any dimension.

For non-separable systems the semiclassical theory breaks down - mainly due to the lack of an explicit functional that maps a given eigenfunction to its nodal count. In this case one may still find the nodal count numerically using for instance variants of the Hoshen-Kopelman algorithm [16]. For two-dimensional systems with a corresponding classical dynamics that shows chaos (this is usually referred to as quantum or wave chaos) such an approach revealed that the limiting distribution is universal. Independent of the details of the system the limiting distribution contracts to a Gaussian located at a universal value $\xi_{\mathrm{u}}$, i.e. $P(\xi)=\delta\left(\xi-\xi_{u}\right)$ [14]. Consistency with Berry's random wave conjecture [17] has also been checked numerically - the conjecture states that eigenfunctions of a chaotic billiard follow the same statistics as the (monochromatic) Gaussian random wave model (a random superposition of plane waves of the same wavelength).

The universality of the nodal count statistics for wave-chaotic systems in two dimensions has been explained in a seminal work by Bogomolny and Schmit [18] who constructed a heuristic parameter-free critical percolation model that predicts the numerical value 
of $\xi_{\mathrm{u}}$ in perfect agreement with numerical calculations and with the Gaussian random wave model (see also [19, 20, 21]). Proving rigorously the implied conjecture that the two-dimensional Gaussian random wave model and wave functions of chaotic billiards are realisations of critical percolation is certainly one of the most challenging open mathematical questions in the field. Indeed a few of the implied properties have already been proven for random spherical harmonics [22]. A related and equally challenging conjecture states that the nodal lines for such systems are a realisation of stochastic Loewner evolution (SLE) [19, 21, 23, 24, 25]. The theoretically known statistical properties of nodal counts in two-dimensional wave-chaotic systems have also been tested thoroughly in experimental settings [26, 27, 28].

Preliminary theoretical and numerical results for two-dimensional systems that are neither separable nor nor fully wave chaotic have been obtained for non-integrable systems with mixed phase space [29] and for integrable systems for which the wave equation is not separable [30]. Especially the latter shows that nodal count statistics in non-separable integrable systems have a high degree of complexity with a few features that resemble either the separable or the wave-chaotic case while new features appear.

In this work we address nodal counts in arbitrary dimensions. Indeed little is known for dimension larger than two. We will focus on the separable case. In Section 2 we derive an asymptotic expression for the normalised nodal count and related it to the geometry of the unit energy shell in action space. In Section 3 we will give a general expression for the limiting nodal count distribution and show that it has some universal properties whose details change with the dimension. In Section 4 the cuboid and the harmonic oscillator are discussed in more detail and the limiting distribution is compared to numerically obtained histograms for finite energy intervals. Eventually we will discuss in Section 5 some generalisations of our results and also comment on the nodal count for wave chaotic systems and random waves in higher dimensions.

\section{Nodal domain distributions for separable systems}

We consider nodal counts for solutions of the wave equation (1) in the case where a separation Ansatz leads to the full solution of the eigenvalue problem. The tools we will apply for the derivation of the nodal counts and of the limiting distribution (4) are EBK quantisation and Poisson summation. The asymptotic limit will be an integral over a region in phase space, and it will involve only classical quantities. We will start with introducing the relevant classical mechanics. We will not try to be as general as possible during the derivation. Rather we will make some assumptions that simplify the derivation and later discuss (see Section 5) which assumptions are essential and which may be relaxed. 


\subsection{EBK quantisation and its implication for nodal counts}

Separability of the wave equation (1) implies that there exist coordinates $\mathbf{q}=\left(q^{1}, \ldots, q^{s}\right)$ which (almost) cover the whole $s$-dimensional manifold $\mathcal{M}$ such that any eigenfunction can be written in a product form

$$
\Phi(\mathbf{q})=\prod_{l=1}^{s} \phi^{(l)}\left(q_{l}\right) .
$$

For such systems semiclassical Einstein-Brillouin-Keller (EBK) quantisation can be performed successfully. The corresponding classical Hamiltonian Mechanics on the phase space $T^{*} \mathcal{M}$ (cotangent bundle to the configuration manifold $\mathcal{M}$ ) is generated by the

$$
H(\mathbf{p}, \mathbf{q})=\sum_{u, v=1}^{s} g^{u v}(\mathbf{q}) p_{u} p_{v}+V(\mathbf{q})
$$

where $p_{l}$ is the conjugate momentum to $q^{l}$, and $g^{u v}$ is the inverse to the metric tensor $g_{u v}$ which defines the squared distance $d s^{2}=\sum_{u, v=1}^{s} g_{u v}(\mathbf{q}) d q^{u} d q^{v}$.

Quantum separability implies that the corresponding Hamiltonian dynamics is integrable. The dynamics is confined to an $s$-dimensional sub-manifold defined by $s$ independent constants of motion $C_{n}(\mathbf{p}, \mathbf{q})=c_{n}$ in phase space that (generically) has the topology of a torus. One introduces the action variables

$$
I_{l}=\frac{1}{2 \pi} \oint_{\mathbf{c}} p_{l} d q^{l}
$$

where the integration is a long the curve in the $p_{l}-q^{l}$ plane where it intersects with the torus defined by the values $\mathbf{c}$ for the constants of motion - the action is proportional to the area enclosed by the torus in that plane. One may perform a canonical transformation to action and angle variables $(\mathbf{p}, \mathbf{q}) \mapsto(\mathbf{I}, \boldsymbol{\theta})$ where $\boldsymbol{\theta}=\left(\theta^{1}, \ldots, \theta^{s}\right)$ are conjugate to the actions $\mathbf{I}=\left(I_{1}, \ldots, I_{s}\right)$. I.e. the phase space is foliated in tori such that a point in phase space is specified by the torus with action variables $\mathbf{I}$ and the position on the torus specified in terms of the $s$ angles $\boldsymbol{\theta}$. As the action variables are constants of motion all angle variable become cyclic variables for the transformed Hamilton function $H(\mathbf{I})$.

We will make the following additional assumptions on the classical Hamiltonian dynamics:

(A1) The potential is non-negative and the classically allowed region $\mathcal{V}_{E}=$ $\{\mathbf{q} \in \mathcal{M}: V(\mathbf{q}) \leq E\}$ is connected and compact. We have stated this assumption in the introduction. This assumption ensures we have a discrete non-negative spectrum.

(A2) There is a one-to-one correspondence between tori in phase space and points $\mathbf{I}$ in action space. This assumption excludes double-well potentials and similar potentials in higher dimensions where action variables can only be defined locally in regions bounded by stationary points and separatrices. 
(A3) A related assumption is the Hamiltonian is a strictly increasing function of all action variables

$$
\omega^{l}(\mathbf{I}) \equiv \frac{\partial H}{\partial I_{l}}>0
$$

Here $\omega^{l}(\mathbf{I})$ is the angular velocity of the angle variable $\theta^{l}$ on the torus defined by $\mathbf{I}$. We also assume that the Hessian matrix $\frac{\partial^{2} H}{\partial I_{l} \partial I_{l}^{\prime}}$ at any point is non-negative.

(A4) Each action takes positive values $I_{l} \geq 0$ and is not bounded from above. This assumption excludes that any of the variables $q^{l}$ in which the wave function separates is cyclic. This is less restrictive than it may appear: for a system with rotational invariance one may reduce the attention either to functions which are even or odd under a reflection with respect to a hyperplane through the axis of rotation.

(A5) We assume that the Hamilton function is a homogeneous function of the actions. For $\lambda>0$ we then have

$$
H(\lambda \mathbf{I})=\lambda^{\alpha} H(\mathbf{I})
$$

where $\alpha>0$ is the degree of homogeneity. This assumption implies that the dynamics in each energy shell is equivalent up to a scaling factor. For free motion on a manifold one has $\alpha=2$, so this assumption is mainly a restriction on the potentials. Note that the harmonic oscillator in any dimension has degree $\alpha=1$.

The above assumptions are not completely independent. Some may be relaxed without distorting our discussion too much (see Section 5).

The EBK spectrum of semiclassical energy eigenvalues is given by

$$
E_{\mathbf{n}}^{\mathrm{EBK}}=H\left(I_{1}=n_{1}+\mu_{1}, \ldots, I_{s}=n_{s}+\mu_{s}\right)
$$

where the quantum numbers $n_{l}=0,1,2, \ldots$ are non-negative integers, and the shifts $\mu_{l}$ are fixed numbers of order unity. E.g. the $s$-dimensional harmonic oscillator has $\mu_{l}=1 / 2$ for all $l$ and free motion on an $s$-dimensional cuboid with Dirichlet boundary conditions has $\mu_{l}=1$. For our discussion the actual value of $\mu_{l}$ is not relevant.

For a given set of quantum numbers the wave function can be written as

$$
\Phi_{\mathbf{n}}(\mathbf{q})=\prod_{l=1}^{s} \phi_{\mathbf{n}}^{(l)}\left(q^{l}\right)
$$

with real functions $\phi_{\mathbf{n}}^{(l)}\left(q^{l}\right)$ of one variable. The corresponding nodal pattern will then have a checker board structure. Each of these functions obeys Sturm's oscillation theorem, i.e. $\phi_{\mathbf{n}}^{(l)}\left(q^{l}\right)$ contains $n_{l}$ nodal points. For the explicit EBK wave functions this is straight forward to show. This implies that the number of nodal domains in the wave function $\Phi_{\mathbf{n}}(\mathbf{q})$ is equal to

$$
\nu_{\mathbf{n}}=\prod_{l=1}^{s}\left(n_{l}+1\right) .
$$

Note that for a degenerate spectrum separability implies a definite and natural choice of preferred basis functions. 


\subsection{The normalised nodal counts and Weyl's law}

In order to find the normalised nodal count $\xi_{N}=\nu_{N} / N$ for a given wave function with quantum numbers $\mathbf{n}=\left(n_{1}, \ldots, n_{s}\right)$ we need to know the spectral counting index $N \equiv N(\backslash)$. An exact ordering of the quantum numbers is a formidable task - in the degenerate case one also needs to make some choice for the order of basis functions with the same energy. In the present context any such order would be fine - as it turns out to leading order one only needs a sufficiently good approximation to the exact counting index as provided by Weyl's law. Indeed the semiclassical approximation we use introduces an error in the ordering which may easily exceed any influence of degeneracies. Weyl's law states that

$$
N(E) \sim \mathcal{V}_{\Gamma} E^{s / \alpha}
$$

gives the leading asymptotic order of the spectral counting index as $E \rightarrow \infty$. Here

$$
\mathcal{V}_{\Gamma}=\int_{\Gamma} d \mathbf{I}
$$

is the volume of the region $\Gamma \equiv\{\mathbf{I}: 0 \leq H(\mathbf{I}) \leq 1\}$ in action space. For a free particle

it is related to the volume $\mathcal{V}_{\mathcal{M}}$ of the manifold by $\mathcal{V}_{\Gamma}=\mathcal{V}_{\mathcal{M}} \frac{\mathcal{V}_{B_{s}}}{(2 \pi)^{s}}$ where $\mathcal{V}_{B_{s}}=\frac{\pi^{s / 2}}{\Gamma\left(\frac{s}{2}+1\right)}$ is the volume of the $s$-dimensional unit ball.

Weyl's law (14) allows us to write the asymptotic expression

$$
\xi_{\mathbf{n}} \sim \frac{\prod_{l=1}^{s}\left(n_{l}+1\right)}{[H(\mathbf{n}+\boldsymbol{\mu})]^{s / \alpha} \mathcal{V}_{\Gamma}} \sim \frac{\prod_{l=1}^{s} n_{l}}{E_{\mathbf{n}}^{s / \alpha} \mathcal{V}_{\Gamma}}+\mathcal{O}\left(E_{\mathbf{n}}^{-1 / \alpha}\right)
$$

for the normalised nodal count. The error estimate on the right side of (16) is based on the homogeneity of the Hamilton function which implies $n_{l} \sim E^{1 / \alpha}$. Expression (16) will serve as the starting point of the derivation of the limiting distribution in section 3 .

Let us now derive Weyl's law (14) in the present setting. This will not only serve us to estimate next-to leading orders in the asymptotic formulas (14) and (16) but also give us an opportunity to introduce further details of the setting. The exact spectral counting function is defined by

$$
N(E)=\#\left\{E_{\mathbf{n}} \leq E\right\}=\sum_{\mathbf{n}} \Theta\left(E-E_{n}\right)
$$

where $\Theta(x)$ is Heaviside's unit step function. Replacing the exact energies by the EBK approximation introduces a small error by shifting the positions of the steps slightly. The error introduced by this shift is much smaller than the fluctuations in the spectral counting function around its mean value and will be neglected. The Poisson summation formula in the form

$$
\sum_{n=0}^{\infty} F(n)=\sum_{M=-\infty}^{\infty} \int_{-1 / 2}^{\infty} e^{2 \pi M x} F(x) d x
$$

and the homogeneity of the Hamilton function asserted by assumption (A5) allow us to write

$$
N(E)=E^{s / \alpha} \sum_{\mathbf{M}} \int_{I_{l}>\frac{\mu_{l}-1 / 2}{E^{1 / \alpha}}} e^{2 \pi i E^{1 / \alpha} \mathbf{M} \cdot \mathbf{I}-2 \pi i \mathbf{M} \cdot \mu} \Theta(1-H(\mathbf{I})) d^{s} \mathbf{I}
$$


On the Nodal Count Statistics for Separable Systems in any Dimension

$$
=\bar{N}(E)+N_{\text {osc }}(E)
$$

where

$$
\bar{N}(E)=E^{s / \alpha}\left(\int_{\Gamma} d^{s} \mathbf{I}+\mathcal{O}\left(E^{-1 / \alpha}\right)\right) \sim E^{s / \alpha} V_{\Gamma}
$$

is the contribution from the non-oscillating integral $M_{1}=M_{2}=\ldots=M_{s}=0$ and $N_{\text {osc }}(E)$ is the sum over all remaining (oscillating) integrals - each being at most of order $E^{(s-1) / \alpha}$. Altogether we have derived Weyl's law (14) and estimated that the sub-leading correction is a factor of order $E^{1 / \alpha}$ smaller than the leading term.

\subsection{The geometry of the energy shell and rescaled actions}

It is worth looking at the geometry of the region $\Gamma$ and the hyper-surface $\partial \Gamma$ in more detail (see Figure 1 for an illustration). Indeed we here deal integrals of the type

$$
\mathcal{I}=\int_{E \cdot \Gamma} f(\mathbf{I}) d^{s} I
$$

with a homogeneous function $f(\mathbf{I})$ (of order $\beta$ ). Here $E \cdot \Gamma=\{\mathbf{I}: H(\mathbf{I}) \leq E\}$ is a scaled version of the region $\Gamma$. Note that $\Gamma$ is compact and convex - compactness follows from assumption (A1) and convexity from the second part of assumption (A3). Indeed, compactness of the classically allowed region $\mathcal{V}_{E}$ implies compactness of the region $\mathcal{W}=\{(\mathbf{p}, \mathbf{q}): H(\mathbf{p}, \mathbf{q}) \leq 1\}$ in phase space because the allowed momenta for any point $\mathbf{q} \in \mathcal{V}(E)$ form a closed $s$-dim ball in the cotangent space $T^{*} \mathcal{M}_{\mathbf{q}}$. Describing the region $\mathcal{W}$ in action-angle variables eventually implies compactness.

Using homogeneity one may reduce the $s$-dimensional integral (22) to an $s-1$ dimensional integral over the $s$-1-dimensional compact surface (unit energy shell) $\partial \Gamma \equiv\{\mathbf{I}: H(\mathbf{I})=1\}$ in momentum space. Note that $\partial \Gamma$ is the non-trivial part of the boundary of the region $\Gamma$ and intersects the hyperplanes $I_{l}=0$ (which are also boundaries of $\Gamma$ ).

This reduction is performed by a substitution to rescaled action variables. The latter are defined by

$$
\mathbf{I}=\varepsilon \mathbf{J}
$$

such that

$$
H(\mathbf{J})=1 .
$$

The rescaled action variables $J_{l}$ are not independent. Assumption (A3) allows us to use the implicit function theorem and solve (24) for

$$
\hat{I}_{s}=Z_{\Gamma}\left(J_{1}, \ldots, J_{s-1}\right) .
$$

We will denote the $s$-1-tuple of rescaled actions that appears as the argument by $\mathbf{J}_{\Omega} \equiv\left(J_{1}, \ldots, J_{s-1}\right)$ such that $\mathbf{J}=\left(\mathbf{J}_{\Omega}, Z_{\Gamma}\left(\mathbf{J}_{\Omega}\right)\right)$. Again using assumption (A2) one can show that the function $Z_{\Gamma}\left(\mathbf{J}_{\Omega}\right)$ is a decreasing of all arguments because

$$
\frac{\partial Z_{\Gamma}}{\partial J_{l}}=-\frac{\omega_{l}\left(\mathbf{J}_{\Omega}, Z_{\Gamma}\left(\mathbf{J}_{\Omega}\right)\right)}{\omega_{s}\left(\mathbf{J}_{\Omega}, Z_{\Gamma}\left(\mathbf{J}_{\Omega}\right)\right)}
$$




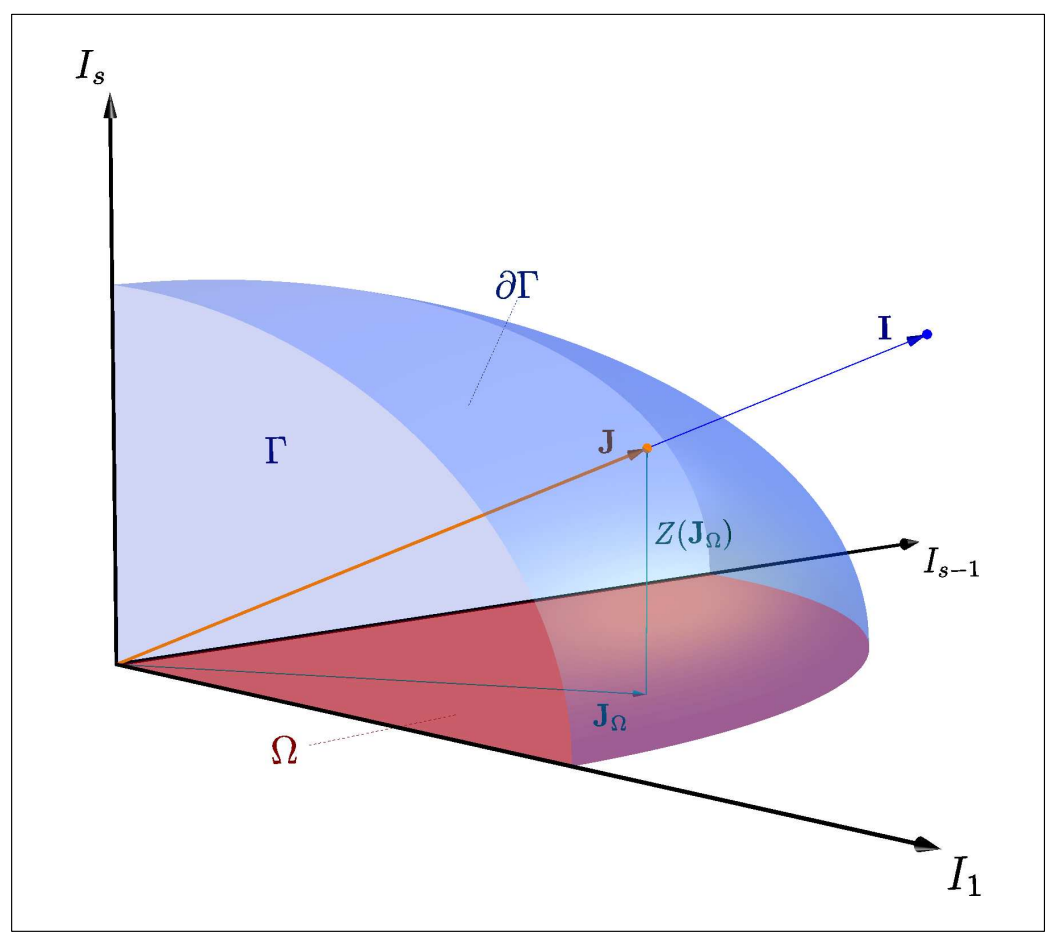

Figure 1. Illustration of the region $\Gamma=\{\mathbf{I}: H(\mathbf{I}) \leq 1\}$ in $s$-dimensional action space. The $s$-1-dimensional hyperplane $I_{s}=0$ is represented by a two-dimensional plane spanned by the $I_{1}$ and $I_{s-1}$ axes in this picture. The illustration also shows the hypersurfaces $\partial \Gamma$ (the unit energy shell, i.e. the level set of the Hamilton function $H(\mathbf{I})=1$ ) and $\Omega$. These are the upper and lower parts of the boundary of $\Gamma$.

A general point in action space with coordinates $\mathbf{I}$ is projected onto the unit energy shell $\partial \Gamma$ where it is represented by the $s$-tuple $\mathbf{J}$.

by the implicit function theorem. The intersection of $\partial \Gamma$ with $J_{s}=Z_{\Gamma}\left(\mathbf{J}_{\Omega}\right)=0$ marks the boundary of the range $\Omega$ of the variables $\mathbf{J}_{\Omega}$. 
Let us now come back to the transformation (23). It implies a change of integration variables from the $s$ unscaled actions $\mathbf{I}$ to the independent $s-1$ scaled actions $\mathbf{J}_{\Omega}$ with values in the region $\Omega$ and a scaling factor $\varepsilon \in[0, E]$. The Jacobean can be calculated straight forwardly and is given by

$$
\mathcal{J}=\varepsilon^{s-1}\left(Z_{\Gamma}\left(\mathbf{J}_{\Omega}\right)-\mathbf{J}_{\Omega} \cdot \nabla_{\mathbf{J}_{\Omega}} Z_{\Gamma}\left(\mathbf{J}_{\Omega}\right)\right)=\varepsilon^{s-1} \frac{\alpha}{\omega_{s}(\mathbf{J})}
$$

where the right hand-side follows from Euler's homogeneous function theorem for the Hamilton function. The Jacobean is thus positive for $\mathbf{J}_{\Omega} \in \Omega$ and $\varepsilon \in(0, E]$. With the shorthand

$$
d \Gamma=\left(Z_{\Gamma}\left(\mathbf{J}_{\Omega}\right)-\mathbf{J}_{\Omega} \cdot \nabla_{\mathbf{J}_{\Omega}} Z_{\Gamma}\left(\mathbf{J}_{\Omega}\right)\right) \prod_{l=1}^{s-1} d J_{l}
$$

we may now rewrite $(22)$ as

$$
\mathcal{I}=\frac{E^{s+\beta}}{s+\beta} \int_{\Omega} d \Gamma f\left(\mathbf{J}_{\Omega}, Z\left(\mathbf{J}_{\Omega}\right)\right) .
$$

For $f \equiv 1$ this implies

$$
\mathcal{V}_{\Gamma}=\frac{1}{s} \int_{\Omega} d \Gamma
$$

It is worth giving a geometrical illustration of the asymptotic nodal count (16). In rescaled action variables $n_{l} \equiv I_{l}=\varepsilon J_{l}$ the normalised nodal counts becomes a ratio

$$
\xi_{\mathbf{n}} \sim \frac{\mathcal{V}\left(\mathbf{J}_{\Omega}\right)}{\mathcal{V}_{\Gamma}}
$$

where

$$
\mathcal{V}\left(\mathbf{J}_{\Omega}\right)=\left(\prod_{l=1}^{s-1} J_{l}\right) Z\left(\mathbf{J}_{\Omega}\right)
$$

is the volume of an $s$-dimensional cuboid in action space with faces parallel to the hyperplanes $I_{l}=0$, and with one corner in the origin and the other on a point $\mathbf{J}=\left(\mathbf{J}_{\Omega}, Z\left(\mathbf{J}_{\Omega}\right)\right)$ on the surface $\partial \Gamma$ (see figure 2 for an illustration). As $\mathcal{V}\left(\mathbf{J}_{\Omega}\right)<\mathcal{V}_{\Gamma}$ we immediately obtain $\xi\left(\mathbf{J}_{\Omega}\right)<1$ which is consistent with Courant's theorem [5]. Since the maximal value of the volume $\mathcal{V}\left(\mathbf{J}_{\Omega}\right)$ is definitely smaller than $\mathcal{V}_{\Gamma}$ the result is also consistent with Pleijel's theorem [6]. Let $\mathbf{J}_{\text {crit }}$ be the values for the rescaled action where $\mathcal{V}\left(\mathcal{J}_{\Omega}\right)$ takes its maximal value for $\mathbf{J}_{\Omega} \in \Omega$. Then $\mathbf{J}_{\text {crit }}$ is a solution of the equations

$$
Z\left(\mathbf{J}_{\Omega}\right)=-J_{l} \frac{\partial Z\left(\mathbf{J}_{\Omega}\right)}{\partial J_{l}} \quad l=1, \ldots, s-1 .
$$

Note that the left hand side is a strictly decreasing function of $J_{l}$ while convexity of $\Gamma$ implies that the right hand side is an increasing function. As a consequence the solution to equation (33) is unique and $\mathcal{V}\left(\mathbf{J}_{\Omega}\right)$ has only one critical point in $\Omega$ which is the global maximum.

In the asymptotic regime $E \rightarrow \infty$ there will be no normalised nodal counts which are larger than the critical value

$$
\xi_{\text {crit }}=\frac{\mathcal{V}\left(\mathbf{J}_{\text {crit }}\right)}{\mathcal{V}_{\Gamma}}<1
$$




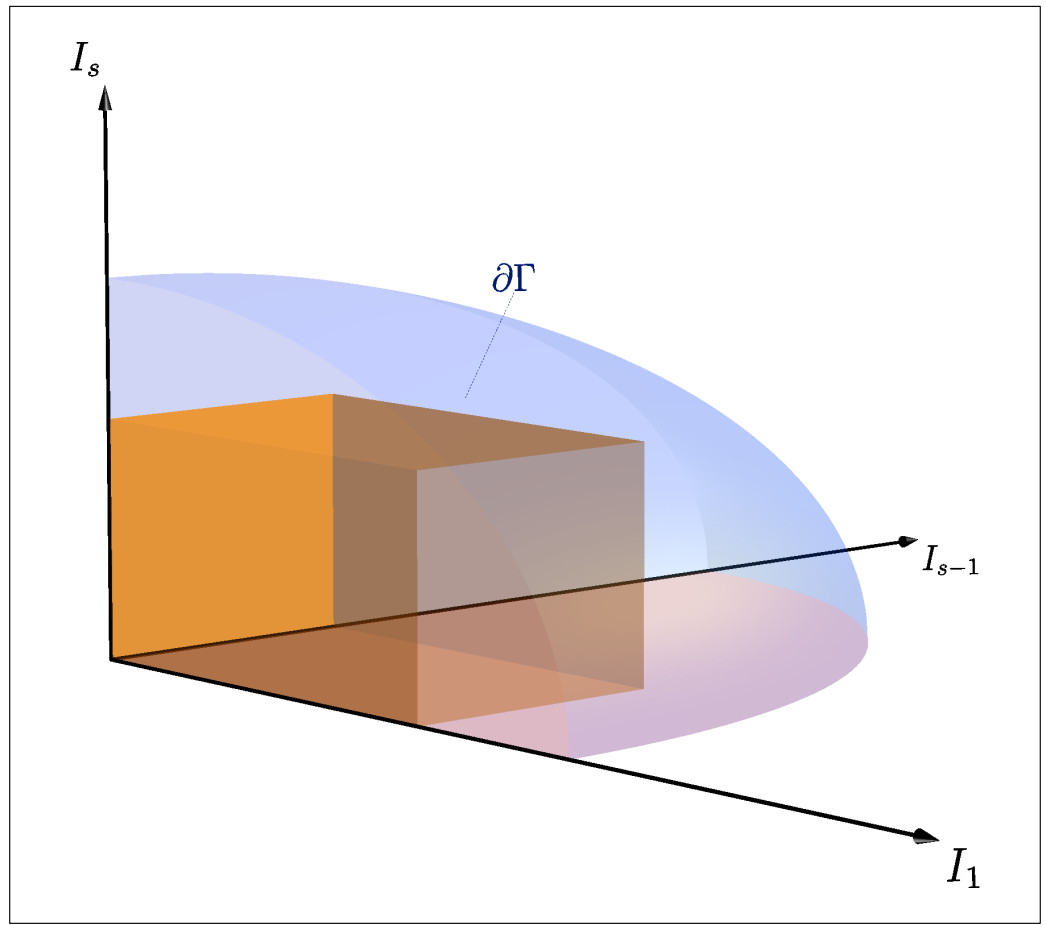

Figure 2. Illustration of the geometric interpretation of the normalised nodal count as a ratio of two volumes $\xi\left(\mathbf{J}_{\Omega}\right)=\mathcal{V}\left(\mathbf{J}_{\Omega}\right) / \mathcal{V}_{\Gamma}<1$. The cuboid of volume $\mathcal{V}\left(\mathbf{J}_{\Omega}\right)$ is inscribed in the region $\Gamma$ with volume $\mathcal{V}_{\Gamma}$. The faces of the cuboid are parallel to the hyper-surfaces $I_{l}=0$ with one corner at the origin and the opposite corner on the surface $\partial \Gamma$.

Simple geometric intuition based on this picture shows that $\xi_{\text {crit }}$ will usually not be very close to either zero or unity for moderate dimensions - in high dimensions one may have $\xi_{\text {crit }} \ll 1$. 


\section{The nodal count distribution and its universal properties}

Let us now consider the nodal domain distribution (3). Poisson summation (18) and an application of $(29)$ then gives $P(\xi)_{I_{g}(E)} \sim P(\xi)+\mathcal{O}\left(E^{-1 / \alpha}\right)$ with the limiting distribution

$$
P(\xi)=\frac{1}{s \mathcal{V}_{\Gamma}} \int_{\Omega} d \Gamma \delta\left(\xi-\frac{\mathcal{V}\left(\mathbf{J}_{\Omega}\right)}{\mathcal{V}_{\Gamma}}\right) .
$$

The limiting distribution above does not depend on the size $g$ of the spectral interval $I_{g}(E)=[E,(1+g) E]$. Note also that $P(\xi)$ is obtained as a weak limit, i.e. in the sense of weak convergence of linear functionals which (together with the fact that the support is always finite) ensures convergence of all moments.

In practice one may consider $P(\xi)_{I_{g}(E)}$ numerically in the form of a histogram (i.e. in a locally averaged form) and these will have some corrections to the limiting distribution - these corrections will depend on the energy $E$, the size $g$ of the spectral interval, and the bin size that has been used for the histogram. As $E \rightarrow \infty$ with $g$ and bin size fixed the fluctuation will become smaller. Indeed one may decrease the bin size moderately as $E$ increases - for convergence to a smooth function one just has to ensure that the number of normalised nodal counts per bin increases indefinitely.

Expression (35) is quite general and we will now turn deriving some universal properties by a close analysis of this expression. In section 2.3 we have already mentioned that there is an upper bound $\xi_{\text {crit }}<1$ to the normalised nodal count. This implies a cut-off for the nodal domain distribution $P(\xi)$ which has its support inside $0 \leq \xi \leq \xi_{\text {crit }}$. Within its support $P(\xi)$ is differentiable. This follows from the fact that $V\left(\mathbf{J}_{\Omega}\right)$ has only one critical point (maximum) in $\Omega$. At $\xi=0$ and $\xi=\xi_{\text {crit }}$ the distribution $P(\xi)$ may have singularities. We will show that the behaviour at the cut-off is mainly governed by the dimension $s$. For $s=2$ one has a square root divergence, for $s=3$ there is a finite step, and for $s \geq 4$ the distribution becomes continuous at $\xi=\xi_{\text {crit }}$ but not smooth.

\subsection{The behaviour of $P(\xi)$ near the cut-off $\xi_{\text {crit }}$.}

For $\xi$ smaller and close to $\xi_{\text {crit }}$ the contribution to $P(\xi)$ depend on the behaviour of $\mathcal{V}\left(\mathbf{J}_{\Omega}\right)$ near its maximal value which it takes at $\mathbf{J}_{\text {crit }}$. Taylor expansion of $\mathcal{V}\left(\mathbf{J}_{\Omega}\right)$ to second order around the maximum gives

$$
\frac{\mathcal{V}\left(\mathbf{J}_{\Omega}\right)}{\mathcal{V}_{\Gamma}}=\xi_{\text {crit }}-\sum_{l, l^{\prime}=1}^{s-1} \mathcal{H}_{l l^{\prime}} \Delta J_{l} \Delta J_{l^{\prime}}+\mathcal{O}\left(\Delta J^{3}\right)
$$

where $\Delta J_{l}=J_{l}-J_{\text {crit }, l}$ and $\mathcal{H}_{l l^{\prime}}$ is a positive definite matrix. From

$$
d \Gamma=\left(s Z\left(\mathbf{J}_{\text {crit }}\right)+\mathcal{O}(\Delta J)\right) \prod_{l=1}^{s} d J_{l}
$$

one obtains

$$
P(\xi) \sim \frac{Z\left(\mathbf{J}_{\text {crit }}\right) \mathcal{V}_{\mathcal{S}^{s-2}}}{2 \mathcal{V}_{\Gamma} \sqrt{\operatorname{det} \mathcal{H}}}
$$


where $\mathcal{V}_{\mathcal{S}^{s-2}}=2 \pi^{(s-1) / 2} / \Gamma((s-1) / 2)$ is the volume of the $s-2$-dimensional sphere. The two dimensional case $s=2$ is included in this analysis, with $\mathcal{V}_{\mathcal{S}^{0}}=2$. In this case $P(\xi)$ diverges $\propto \frac{1}{\sqrt{\xi_{\text {crit }}-\xi}}$ as was shown before in [14]. When $s=3$ we observe that $P(\xi) \rightarrow$ const $>0$ as $\xi \rightarrow \xi_{\text {crit }}$ from below. For $s \geq 4$ we have $P(\xi) \propto\left(\xi_{\text {crit }}-\xi\right)^{(s-3) / 2} \rightarrow 0$ such that $P(\xi)$ is continuous at $\xi=\xi_{\text {crit }}$.

\subsection{The behaviour of $P(\xi)$ near $\xi=0$.}

Now, we will study the behaviour of $P(\xi)$ near $\xi=0$. For $s=2$ it is not difficult to show that

$$
\lim _{\xi \rightarrow 0^{+}} P(\xi)=\frac{1}{2} \lim _{J_{1} \rightarrow 0} \frac{Z\left(J_{1}\right)-J_{1} Z^{\prime}\left(J_{1}\right)}{Z\left(J_{1}\right)+J_{1} Z^{\prime}\left(J_{1}\right)}+\frac{1}{2} \lim _{Z \rightarrow 0} \frac{J_{1}(Z)-Z J_{1}^{\prime}(Z)}{J_{1}(Z)+Z J_{1}^{\prime}(Z)}=1
$$

where $J_{1}(Z)$ is the inverse function of $Z\left(J_{1}\right)$.

For the rest of this section we keep our focus on $s \geq 3$. Note that the $\delta$-function $\delta\left(\xi-\mathcal{V}\left(\mathbf{J}_{\Omega}\right) / \mathcal{V}_{\Gamma}\right)$ in expression (35) for $\xi<\xi_{\text {crit }}$ reduces the integral to an $s-2$ dimensional integral over the level surfaces of $\mathcal{V}\left(\mathbf{J}_{\Omega}\right)$. These are closed deformations of an $s-2$-dimensional sphere. For our present purpose it is useful to write

$$
\begin{aligned}
& d \Gamma=s d \Gamma_{1}+d \Gamma_{2} \\
& d \Gamma_{1}=Z\left(\mathbf{J}_{\Omega}\right) \prod_{l=1}^{s-1} d J_{l} \\
& d \Gamma_{2}=-\frac{Z\left(\mathbf{J}_{\Omega}\right)}{\mathcal{V}\left(\mathbf{J}_{\Omega}\right)}\left[\mathbf{J}_{\Omega} \cdot \nabla_{\mathbf{J}_{\Omega}} \mathcal{V}\left(\mathbf{J}_{\Omega}\right)\right] \prod_{l=1}^{s-1} d J_{l}
\end{aligned}
$$

We will show below that $d \Gamma_{2}$ does not give a contribution to the nodal count distribution which then reduces to

$$
P(\xi)=\frac{1}{\mathcal{V}_{\Gamma}} \int d \Gamma_{1} \delta\left(\xi-\frac{\mathcal{V}\left(\mathbf{J}_{\Omega}\right)}{\mathcal{V}_{\Gamma}}\right)=\int_{S_{\xi}} \frac{Z\left(\mathbf{J}_{\Omega}\right)}{\left|\nabla_{\mathbf{J}_{\Omega}} \mathcal{V}\left(\mathbf{J}_{\Omega}\right)\right|} d S_{\xi}
$$

where $d S_{\xi}$ is the surface volume (area) element of the surface

$$
S_{\xi}=\left\{\mathbf{J}_{\Omega}: \mathcal{V}\left(\mathbf{J}_{\Omega}\right)=\xi \mathcal{V}_{\Gamma}\right\}
$$

In order to show that the corresponding integral over $d \Gamma_{2}$ vanishes one may start with

$$
\int_{\Omega} d \Gamma_{2} \delta\left(\xi-\frac{\mathcal{V}\left(\mathbf{J}_{\Omega}\right)}{\mathcal{V}_{\Gamma}}\right)=-\int_{S_{\xi}} \frac{\mathbf{J}_{\Omega} \cdot \mathbf{n}}{\prod_{l=1}^{s-1} J_{l}} d S_{\xi}
$$

where $\mathbf{n}=\nabla_{\mathbf{J}_{\Omega}} \mathcal{V}\left(\mathbf{J}_{\Omega}\right) /\left|\nabla_{\mathbf{J}_{\Omega}} \mathcal{V}\left(\mathbf{J}_{\Omega}\right)\right|$ is the unit normal vector on the surface $S_{\xi}$. Gauß' theorem turns this into a volume integral over the region $\mathcal{V}\left(\mathbf{J}_{\Omega}\right)>\xi \mathcal{V}_{\Gamma}$ enclosed by the surface. The corresponding integrand is the divergence of the vector $\frac{\mathbf{J}_{\Omega}}{\prod_{l=1}^{s-1} J_{l}}$ which vanishes identically which proves that equation (41) is correct.

For $\xi \rightarrow 0^{+}$the surface $S_{\xi}$ approaches the boundary $\partial \Omega$ of $\Omega$. From (41) we see that the contributions from a volume element $d S_{\xi}$ carry a weight $Z\left(\mathbf{J}_{\Omega}\right) /\left|\nabla_{\mathbf{J}_{\Omega}} \mathcal{V}\left(\mathbf{J}_{\Omega}\right)\right|$, so it will be dominated by any critical points where $\left|\nabla_{\mathbf{J}_{\Omega}} \mathcal{V}\left(\mathbf{J}_{\Omega}\right)\right|=0$ close to $S_{\xi}$. Indeed there are such critical points on the boundary $\partial \Omega$ and they coincide with the set of 
points where $\partial \Omega$ is not smooth. These are the $s-3$-dimensional intersections of any 2 hyperplanes $J_{l}=0$ or of one such hyperplane with $Z\left(\mathbf{J}_{\Omega}\right)=0$. All of these are saddle points.

For $s=3$ the saddles are isolated at the three corners of $\partial \Omega$. The Hessian at these saddle points is not degenerate.

For $s \geq 4$ the saddles are not isolated and the Hessian is degenerate. The saddles form continuous surfaces and where they intersect the suppression $\left|\nabla_{\mathbf{J}_{\Omega}} \mathcal{V}\left(\mathbf{J}_{\Omega}\right)\right|$ close to the intersection is enhanced by the combined effect of two or more intersecting saddle point surfaces.

The $s$ corners $\mathbf{J}^{(c)}(c=0, \ldots, s-1)$ of $\Omega$ thus dominate the $P(\xi)$ for $\xi \rightarrow 0^{+}$(see figure 3 for an illustration). Explicitly the corners are given by the origin $\mathbf{J}^{(0)}$ and the $s-1$ points $\mathbf{J}^{(c)}$ where $Z\left(\mathbf{J}_{\Omega}\right)=0$ intersects with the $s-2$ hyperplanes of the form $J_{l}=0$ where $l \in\{1, \ldots, s-1\}-\{c\}$. At the corners the lowest order of a derivative which does not vanish identically is $(s-1)$. We will show that this leads to a divergence of the nodal count distribution $\lim _{\xi \rightarrow 0^{+}} P(\xi)=\infty$.

We may focus on the leading contribution from the corner at the origin which dominates the distribution for small $\xi$ - indeed the weight $Z\left(\mathbf{J}_{\Omega}\right) /\left|\nabla_{\mathbf{J}_{\Omega}} \mathcal{V}\left(\mathbf{J}_{\Omega}\right)\right|$ suppresses the contribution at the corners due to the factor $Z\left(\mathbf{J}_{\Omega}\right)$ which is zero for all corners apart from the origin.

In order to derive the contribution from the origin let us start by expanding enumerator and denominator of the weight $Z\left(\mathbf{J}_{\Omega}\right) /\left|\nabla_{\mathbf{J}_{\Omega}} \mathcal{V}\left(\mathbf{J}_{\Omega}\right)\right|$ independently. For the denominator one has $\left|\nabla_{\mathbf{J}_{\Omega}} \mathcal{V}\left(\mathbf{J}_{\Omega}\right)\right|=\mathcal{O}\left(\Delta \mathbf{J}_{\Omega}^{s-1}\right)$. The enumerator $Z\left(\mathbf{J}_{\Omega}\right)$ however remains finite $Z\left(\mathbf{J}_{0}\right)=Z_{0}>0$ near the origin. Now consider the contribution

$$
P(\xi) \sim \frac{Z_{0}}{\mathcal{V}_{\Gamma}} \int_{C} \delta\left(\xi-\frac{Z_{0} \prod_{l=1}^{s-1} J_{l}}{\mathcal{V}_{\Gamma}}\right) \prod_{l=1}^{s-1} d J_{l}
$$

from a small region $C$ that contains the origin. We have used $Z\left(\mathbf{J}_{\Omega}\right) \sim Z_{0}$ with corrections $\mathcal{O}\left(\mathbf{J}_{\Omega}\right)$. The calculation is simplified if we take $C$ as an $s$-1-dimensional cuboid with side lengths $a_{l}$

$$
C=\left\{\mathbf{J}_{\Omega}: 0 \leq J_{l} \leq a_{l}, l=1, \ldots, s-1\right\} .
$$

The actual values of the side lengths $a_{l}$ will not enter the leading order which implies that we have a true corner phenomenon and that the leading asymptotic order of integral does not depend on the details of region $C$.

In order to perform the integration set

$$
\zeta=\frac{Z_{0} \prod_{l=1}^{s-1} J_{l}}{\mathcal{V}_{\Gamma}}
$$

and transform coordinates $\mathbf{J}_{\Omega} \rightarrow\left(J_{1}, \ldots, J_{s-2}, \zeta\right)$. The $\zeta$-integral can be performed and leaves

$$
P(\xi) \sim \int_{0}^{a_{1}} \frac{d J_{1}}{J_{1}} \ldots \int_{0}^{a_{s-2}} \frac{d J_{s-2}}{J_{s-2}} \Theta\left(J_{s-2}-\frac{\mathcal{V}_{\Gamma} \xi}{a_{s-1} Z_{0} \prod_{l=1}^{s-3} J_{l}}\right)
$$




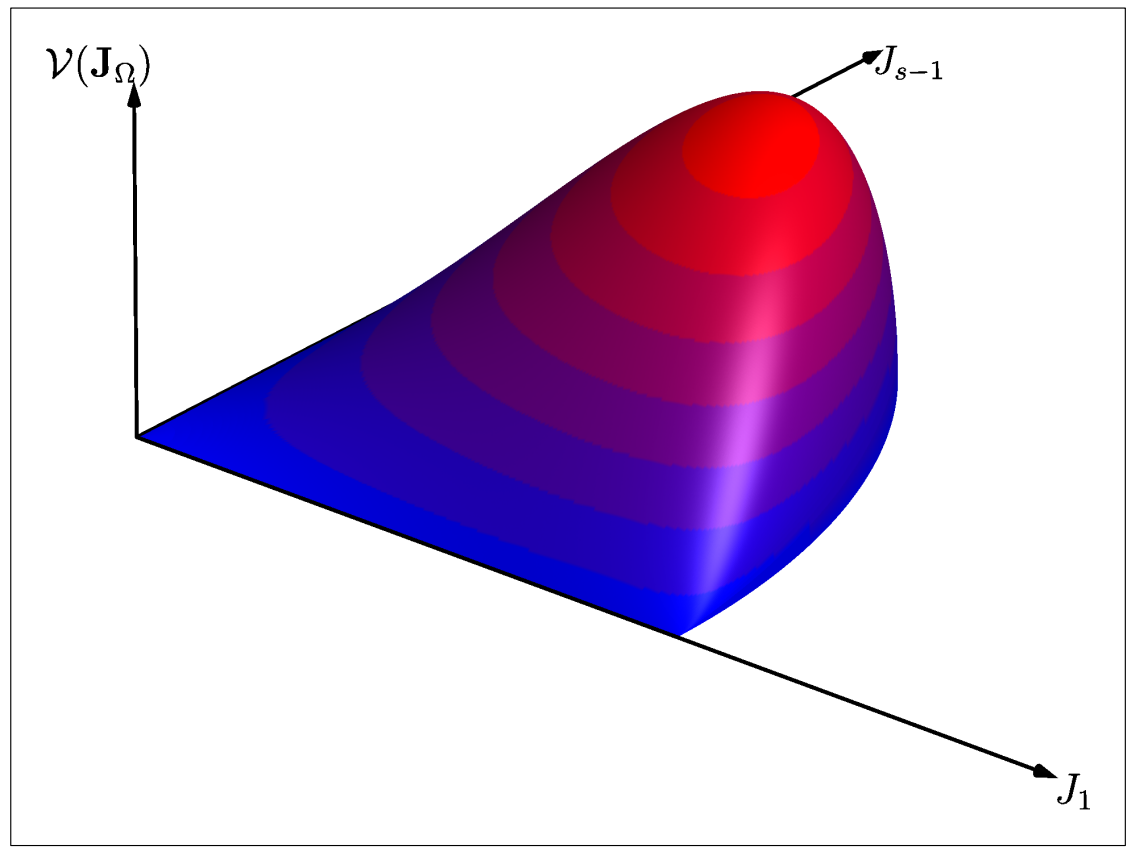

Figure 3. Illustration of the graph of the function $\mathcal{V}(\Gamma)$ over the region $\Omega$. For $s=3$ this illustration is exact and one can see three saddles in the corners of the region $\Omega$. Near these corners, especially near the one at the origin, the values of $\mathcal{V}(\Gamma)$ are strongly suppressed.

For $s>3$ one the illustration has to be taken with some care as it represents an $s-1$ dimensional plane by a two-dimensional - the strong suppression is actually enhanced in this case.

where the factors $J_{l}^{-1}$ stem from the Jacobean. This integral can be solved iteratively using

$$
\int_{0}^{a} \frac{d x}{x}(\log x)^{l} \Theta(x-b)=\Theta(a-b) \frac{(\log a)^{l+1}-(\log b)^{l+1}}{l+1}
$$


and gives the leading contribution

$$
P(\xi) \sim \frac{(-\log \xi)^{s-2}}{(s-2) !} .
$$

Note that the leading order corrections to this depend on the lengths $a_{l}$ which implies that a global approach is necessary to evaluate the next-to-leading order of $P(\xi)$ as $\xi \rightarrow 0^{+}$. We see that the leading order diverges as an $s-2$-th power of a logarithm with a universal constant $1 /(s-2)$ !. Any system dependent features can only enter at next-to-leading order.

\subsection{Monotonicity}

For $s=2$ one can check directly that $P(\xi)$ is a strictly increasing function for $0<\xi<\xi_{\text {crit }}$. Indeed (41) is valid for $s=2$ and gives

$$
P(\xi)=\frac{Z\left(J_{1,-}\right)}{Z\left(J_{1,-}\right)+Z^{\prime}\left(J_{1,-}\right) J_{1,-}}-\frac{Z\left(J_{1,+}\right)}{Z\left(J_{1,+}\right)+Z^{\prime}\left(J_{1,+}\right) J_{1,+}}
$$

where $J_{1,-}<J_{1,+}$ are the two solutions of $\xi=J_{1} Z\left(J_{1}\right)$. Note that both terms in (50) are positive. Differentiation of the first term yields

$$
\frac{J_{1,-} Z^{\prime}\left(J_{1,-}\right)^{2}-Z\left(J_{1,-}\right) Z^{\prime}\left(J_{1,-}\right)-J_{1,-} Z\left(J_{1,-}\right) Z^{\prime \prime}\left(J_{1,-}\right)}{\left(Z\left(J_{1,-}\right)+Z^{\prime}\left(J_{1,-}\right) J_{1,-}\right)^{2}} \frac{d J_{1,-}}{d \xi}>0
$$

because $\frac{d J_{1,-}}{d \xi}>0$. Analogously the derivative of the second term gives a positive contribution because $\frac{d J_{1,+}}{d \xi}<0$.

For $s \geq 3$ our calculations above imply that $P(\xi)$ is a decreasing function in a neighbourhood of $\xi=0$. For $s \geq 4$ we have also shown that $P(\xi)$ is a decreasing function near $\xi=\xi_{\text {crit }}$ (for $s=3$ our results are consistent with a decreasing function). This suggests that $P(\xi)$ may be a decreasing function over its full support $0<\xi<\xi_{\text {crit }}$ for $s \geq 3$. Such a conjecture is supported by all example calculations that we have performed - however we have not been able to prove it.

\section{Two simple examples: the harmonic oscillator and the cuboid}

Let us now illustrate our results with a few examples that allow for more explicit treatment.

\subsection{The s-dimensional harmonic oscillator}

For a harmonic oscillator the Hamilton function is linear in the action variables

$$
H(\mathbf{I})=\sum_{l=1}^{s} \omega_{l} I_{l}
$$

The unit energy shell $\partial \Gamma$ in action space is then described by the function

$$
J_{s} \equiv Z\left(\mathbf{J}_{\Omega}\right)=1-\frac{1}{\omega_{s}} \sum_{l=1}^{s-1} \omega_{l} J_{l}
$$


The volume of the region $\Gamma$ is $\mathcal{V}_{\Gamma}=\frac{1}{s ! \prod_{l=1}^{s} \omega_{l}}$.

From $\mathcal{V}\left(\mathbf{J}_{\Omega}\right)=Z\left(\mathbf{J}_{\Omega}\right) \prod_{l=1}^{s-1} J_{l}$ one finds its maximum value at $J_{\text {crit }, l}=\frac{1}{s \omega_{l}}$ such that $\mathcal{V}\left(\mathbf{J}_{\text {crit }}\right)=\frac{1}{s^{s} \prod_{l=1}^{s} \omega_{l}}$. This implies the critical value

$$
\xi_{\mathrm{crit}, s}=\frac{s !}{s^{s}}
$$

for the normalised nodal count. Note that the individual frequencies do not enter. Indeed the complete nodal count distribution with $s$ degrees of freedom does not depend on the frequencies and can be expressed as

$$
\begin{aligned}
P_{s}(\xi)=s ! & \int_{\sum_{l=1}^{s-1} J_{l} \leq 1}\left(1-\sum_{l=1}^{s-1} J_{l}\right) \times \\
& \times \delta\left(\xi-s !\left(1-\sum_{l=1}^{s-1} J_{l}\right) \prod_{l=1}^{s-1} J_{l}\right) \prod_{l} d J_{l} .
\end{aligned}
$$

For $s=2$ this integral has the explicit form

$$
P_{2}(\xi)=(1-2 \xi)^{-1 / 2} \quad \text { for } \xi<1 / 2 .
$$

For arbitrary $s$ one may evaluate all positive integer moments

$$
\left\langle\xi^{m}\right\rangle_{s} \equiv \int_{0}^{\xi_{\mathrm{crit}, s}} \xi^{m} P_{s}(\xi) d \xi=\frac{s !^{m+1}(m !)^{s}(m+1)}{(s(m+1)) !} .
$$

See Figure 4 for the graph of the limiting distribution (56) for $s=2,3,4$ together with numerical data obtained for finite energy intervals.

\subsection{The s-dimensional cuboid}

We consider the free particle in an $s$-dimensional cuboid (rectangular box) with side lengths $a_{l}(l=1, \ldots, s)$. With Dirichlet conditions on the boundary of the box one obtains exact energy eigenvalues

$$
E_{\mathbf{n}}=\pi^{2} \sum_{l=1}^{s} \frac{n_{l}^{2}}{a_{l}^{2}}
$$

where the quantum numbers $n_{l}$ run over positive integers. The corresponding classical Hamilton function $H(\mathbf{I})=\pi^{2} \sum_{l=1}^{s} \frac{I_{l}^{2}}{s_{l}^{2}}$ is homogeneous of order $\alpha=2$. The unit energy shell $\partial \Gamma$ is given in terms of the function

$$
J_{s} \equiv Z\left(\mathbf{J}_{\Omega}\right)=\frac{a_{s}}{\pi} \sqrt{1-\pi^{2} \sum_{l=1}^{s-1} \frac{J_{l}^{2}}{a_{l}^{2}}} .
$$

The volume of the region $\Gamma$ is $\mathcal{V}_{\Gamma}=\frac{1}{\pi^{s / 2} 2^{s-1} s \Gamma(s / 2)} \prod_{l=1}^{s} a_{l}$.

The maximal volume $\mathcal{V}\left(\mathbf{J}_{\Omega}\right)$ of a cube touching the unit energy shell is given by $\mathcal{V}\left(\mathbf{J}_{\text {crit }}\right)=\frac{1}{\pi^{s} s^{s / 2}} \prod_{l=1}^{s} a_{l}$ (where $\left.J_{\text {crit }, l}=\frac{a_{l}}{s^{1 / 2} \pi}\right)$. One thus finds the critical value

$$
\xi_{\text {crit }, s}=\frac{2^{s-1} s \Gamma(s / 2)}{\pi^{s / 2} s^{s / 2}}
$$



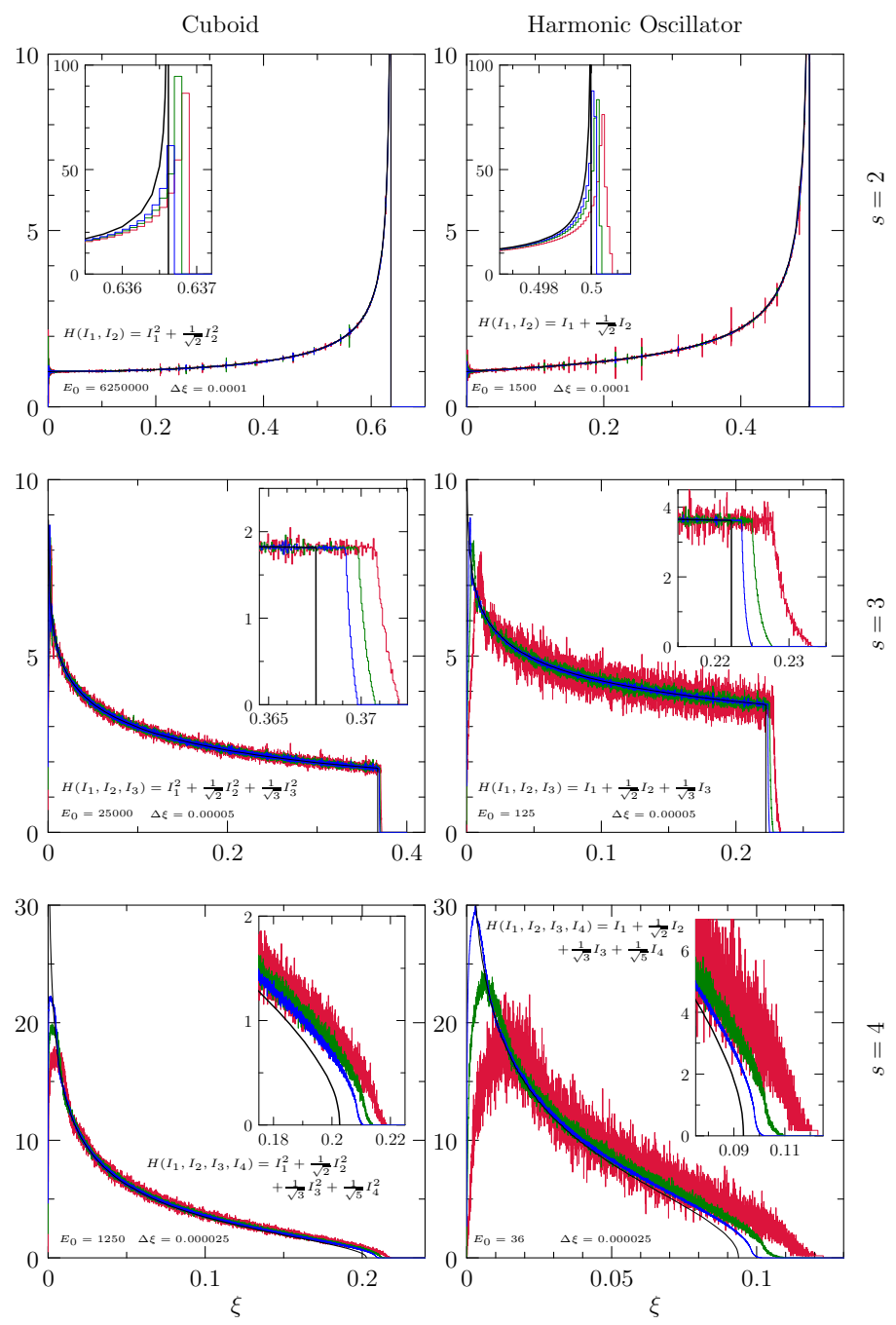

Figure 4. Nodal count distributions for the harmonic oscillator (right column) and the cuboid (left column) for $s=2$ (first row), $s=3$ (second row), and $s=4$ (third row). The black lines correspond to the limiting distributions $P_{s}(\xi)$. The red, green, and blue lines are numerically obtained histograms of the nodal count distribution at finite energy intervals: red line $\left[E_{0}, 2 E_{0}\right]$, green line $\left[2 E_{0}, 4 E_{0}\right]$, blue line $\left[4 E_{0}, 8 E_{0}\right]$. The chosen values for $E_{0}$ are given in the corresponding graphs together with the chosen system parameters (the Hamiltonian), and the bin size $\Delta \xi$ that has been used for the histograms. In each case normalised nodal counts have been obtained for the lowest 50 to 80 million eigenfunctions.

The insets magnify the graph near the critical cut-off value $\xi_{\text {crit }}$. Overall the numerically obtained histograms are consistent with the (weak) convergence to the limiting distribution. 
above which the limiting nodal domain distribution vanishes. Similarly to the harmonic oscillator the critical value and the limiting distribution do not depend on the detailed system parameters such as the side lengths. Indeed the limiting distribution may be written as

$$
\begin{aligned}
P_{s}(\xi)= & \frac{2^{s-1} s \Gamma(s / 2)}{\pi^{s / 2}} \int_{\sum_{l=1}^{s-1} J_{l}^{2}<1}\left(1-\sum_{l=1}^{s-1} J_{l}^{2}\right)^{1 / 2} \times \\
& \delta\left(\xi-\frac{2^{s-1} s \Gamma(s / 2)}{\pi^{s / 2}}\left(1-\sum_{l=1}^{s-1} J_{l}^{2}\right)^{1 / 2} \prod_{l=1}^{s-1} J_{l}\right) \prod_{l=1}^{s-1} d J_{l} .
\end{aligned}
$$

For $s=2$ this reduces to

$$
P_{2}(\xi)=\left(1-\frac{\pi^{2} \xi^{2}}{4}\right)^{-1 / 2} \quad \text { for } \xi<\frac{2}{\pi} .
$$

See Figure 4 for graphs of the distribution for $s=2,3,4$ together with numerically obtained histograms for finite energy intervals.

\section{Discussion}

We have derived an expression for the limiting nodal count distribution in the case where the wave equation is separable, and we have extracted some universal features of this distribution. While we have formally limited the scope with the assumptions (A1) to (A5) many standard examples as the harmonic oscillator or the particle in a cubic box obey all of these conditions. For some other examples which do not obey all of the conditions it is straight forward to generalize our derivations. For instance a particle in a spherical box does not obey assumption (A4) as action variables that correspond to angular momenta are not bounded from below. In this case the wave function separates in variables, some of which are cyclic. The derivation of a limiting distribution follows in full analogy once the expression for the nodal count in terms of quantum numbers is adapted and the Poisson summation is performed over the corresponding set of quantised action variables. Indeed most of our assumptions are purely technical and can be relaxed if necessary - though relaxing condition (A3) may imply that there are additional local maxima of the volume $\mathcal{V}\left(\mathbf{J}_{\Omega}\right)$ which may lead to further singularities within the support of the limiting nodal domain distribution. Also assumption (A5) that the Hamiltonian is a homogeneous function can be relaxed to a certain degree. Indeed it is only needed that the energy shell at high energies can be described asymptotically by a homogeneous function.

It would certainly be interesting to compare our results to nodal count distributions of non-separable or wave-chaotic systems in dimensions larger than two. In any dimension one may try to obtain nodal counts numerically by using a corresponding adaptation of the Hoshen-Kopelman algorithm [16] and apply it to numerically obtained eigenfunctions. Berry's conjecture [17] states that highly excited chaotic eigenfunctions 
can be simulated by a Gaussian random wave ensemble. In this ensemble the wave function is given by

$$
\Phi_{\mathrm{RWM}}(\mathbf{q})=\operatorname{Re} \sqrt{\frac{2}{N}} \sum_{j=1}^{N} e^{i k \mathbf{n}_{\mathbf{j}} \cdot \mathbf{q}+i \phi_{j}}
$$

where $\mathbf{n}_{\mathbf{j}}$ are uniformly distributed on a unit $(s-1)$-sphere and the phases $\phi_{j}$ are equidistributed on $[0,2 \pi)$. On dimensional grounds one expects that the number of nodal domain in a given region of the random wave is proportional to the volume of the region. For two-dimensional random waves this has been checked and it is consistent with the critical percolation conjecture [18]. We tried to check this in three dimensions by finding the number of nodal domains of random waves inside a cube of side length $a$ (at fixed wave number $k=1$ ). The artificial boundary of the cube leads to nodal domains which intersect the boundary - indeed we have found that all nodal domains in our numerical approach were intersecting the boundary and that the nodal count is proportional to $a^{2}$ rather than $a^{3}$. This scaling is expected on dimensional grounds for the number of nodal domains which intersect the boundary. However we were not able to increase the side length beyond $a=100$ (about 16 wave lengths) on a standard desktop and we have just looked at a few hundred realisations. We cannot exclude that interior nodal domains (those which do not touch the artificial boundary) start to appear in much larger cubes and eventually dominate the nodal count. We can say however that any crossover from $a^{2}$ (boundary dominated) to $a^{3}$ (bulk dominated) would have to occur at considerably higher side lengths for which applying our numerical algorithm is beyond the power of standard desktop computers.

Our numerical findings do confirm the basic expectation that the universality of a critical percolation model does not apply in the three dimensional case. For instance we find that the volume of the largest nodal domain scales linearly with the volume of the cubea clear indication that one is inside the (non-universal) percolating regime (a non-trivial exponent is expected at the percolation transition). We hope that future research will shed more light on the nodal sets and nodal counts of wave-chaotic systems in dimensions $s \geq 3$ as well as in the corresponding random-wave models.

\section{References}

[1] E.F.F Chladni, Die Akustik (Breitkopf and Härtel, Leipzig, 1802).

[2] J.C.F. Sturm, Sur les équations différentielles linéaires du second ordre, J. Math. Pures et Appl. de Liouville 1, 106 (1836); Sur une classe d'équations différentielles partielles, J. Math. Pures et Appl. de Liouville 1, 375 (1836).

[3] G. Berkolaiko, A lower bound for nodal count on discrete and metric graphs, Commun. Math. Phys. 278, 803 (2007).

[4] P. Schapotschnikow, Eigenvalue and nodal properties on quantum graph trees, Waves in Random and Complex Media 16, 167 (2006).

[5] R. Courant, Ein allgemeiner Satz zur Theorie der Eigenfunktionen selbstadjungierter Differentialausdrücke, Nachrichten von der Gesellschaft der Wissenschaften zu Gttingen, MathematischPhysikalische Klasse (Göttingen, 13 July 1923), 81 (1923). 
[6] Å. Pleijel, Remarks on Courant's nodal line theorem, Comm. Pure Appl. Math. IX, 543 (1956).

[7] S. Gnutzmann, U. Smilansky, N. Søndergaard, Resolving isospectral drums by counting nodal domains, J. Phys. A 38, 8921 (2005).

[8] S. Gnutzmann, P. Karageorge, U. Smilansky, Can One Count the Shape of a drum?, Phys. Rev. Lett. 97, 090201 (2006).

[9] J. Brüning, D. Klawonn, C. Puhle Comment on, Resolving isospectral drums by counting nodal domains, J. Phys. A 40, 15143 (2007).

[10] S. Gnutzmann, P. Karageorge, U. Smilansky, A trace formula for the nodal count sequence Towards counting the shape of separable drums, Eur. Phys. J. Special Topics 145, 217 (2007)

[11] P.D. Karageorge, U. Smilansky, Counting nodal domains on surfaces of revolution, J. Phys. A 41, 205102 (2008).

[12] D. Klawonn, Inverse Nodal Problems, J. Phys. A 42, 175209 (2009).

[13] J. Brüning, D. Fajman, On the nodal count for flat tori, Comm. Math. Phys., (2012) in press.

[14] G. Blum, S. Gnutzmann, U. Smilansky, Nodal Domain Statistics: A Criterion for Quantum Chaos, Physical Review Letters, 88, 114101 (2002).

[15] U. Smilansky, R. Sankaranarayanan, Nodal domain distribution of rectangular drums, Proceedings of National Conference on Nonlinear Systems and Dynamics (Feb. 24-26, 2005), Aligarh Muslim University, India (2005).

[16] J. Hoshen, R. Kopelman, Percolation and cluster distribution: I. Cluster multiple labeling technique and critical concentration algorithm, Phys. Rev. B 14, 3438 (2006)

[17] M.V. Berry, Regular and irregular semiclassical wave functions, J. Phys. A 1020832091 (1977).

[18] E. Bogomolny, C. Schmit, Percolation Model for Nodal Domains of Chaotic Wave Functions Phys. Rev. Lett. 88, 114102 (2002).

[19] J.P. Keating, F. Mezzadri, A.G. Monastra, Nodal domain distributions for quantum maps, J. Phys. A 36, L53 (2003).

[20] G. Foltin, S. Gnutzmann, U. Smilansky, The morphology of nodal lines: random waves versus percolation J. Phys. A 37, 11363 (2004).

[21] E. Bogomolny, C. Schmit, Random wavefunctions and percolation, J. Phys. A 40, 14033 (2007).

[22] F. Nazarov, M. Sodin, On the Number of Nodal Domains of Random Spherical Harmonics American Journal of Mathematics 131, 1337 (2009).

[23] J.P. Keating, J. Marklof, I.G. Williams, Nodal Domain Statistics for Quantum Maps, Percolation and SLE, Phys. Rev. Lett. 97, 034101 (2006).

[24] E. Bogomolny, R. Dubertrand, C. Schmit, SLE description of the nodal lines of random wave functions J. Phys. A 40, 381 (2007).

[25] J.P. Keating, J. Marklof, I.G. Williams, Nodal domain statistics for quantum chaotic maps, New Journal of Physics 10, 083023 (2008).

[26] N. Savytskyy, O. Hul, L. Sirko, Experimental investigation of nodal domains in the chaotic microwave rough billiard Phys. Rev. E 70, 056209 (2004).

[27] O. Hul, N. Savytskyy, O. Tymoshchuk, S. Bauch, L. Sirko, Investigation of nodal domains in the chaotic microwave ray-splitting rough billiard, Phys. Rev. E 72, 066212 (2005).

[28] U. Kuhl, R. Höhmann, H.-J. Stöckmann, S. Gnutzmann, Nodal domains in open microwave systems, Phys. Rev. E. 75, 036204 (2007).

[29] H. Aiba, T. Suzuki, Nodal Domain Distribution for a Nonintegrable Two-Dimensional Anharmonic Oscillator, Phys. Rev. E 72, 066214 (2005).

[30] A. Aronovitch, R. Band, D. Fajman, S. Gnutzmann, Nodal domains of a non-separable problemthe right-angled isosceles triangle, J. Phys. A 45, 085209 (2012). 